\title{
Design and concept validation of the new solid tungsten divertor for ASDEX Upgrade
}

\author{
A. Herrmann, H. Greuner, N. Jaksic, B. Böswirth, F. Reimold, A. Scarabosio, S. Vorbrugg, \\ M. Wischmeier, and the ASDEX Upgrade team
}

\author{
Max-Planck-Institut für Plasmaphysik, EURATOM Association, D-85748 Garching, Germany
}

\begin{abstract}
Div-III, a divertor with solid tungsten target tiles for ASDEX Upgrade is designed and tested and will be installed in 2013. It is a further step in exploring tungsten as material for plasma facing components. It avoids the restrictions of tungsten coatings on graphite and realizes an operation range up to 50 MJ energy removing capability in the outer divertor. In addition, it allows physics investigation such as erosion and deuterium retention as well as effects of castellation and target tilting. The design of the target itself and the attachment was optimized with FE-analysis and was intensively high heat tested up to a double overload. Cyclic tests reveal that the target and the attachment can be operated with the design load of $50 \mathrm{MJ}$ without any damage. Even a twofold overload results in local recrystallization and minor cracks but the targets did not fail during operation. The redesign of the divertor structure was used to increase the conductance between the cryo-pump and the divertor region. The impact of the changed pumping efficiency was investigated with SOLPS/Eirene modeling. The modelling results are an indication for an easier access to lower SOL densities as expected for a higher pumping efficiency in the main chamber.
\end{abstract}

Keywords: ASDEX Upgrade, Divertor, Tungsten, FEM, GLADIS, Heat load

\section{Introduction}

ASDEX Upgrade (AUG) is a mid-size tokamak fusion experiment that was stepwise transformed from a carbon to a tungsten first wall experiment. Starting with the experimental campaign 2007 AUG was operated as a full tungsten experiment. It could be shown that tungsten and ITER like plasma performance are compatible as long as the central heating of the plasma is high enough to suppress tungsten accumulation in the core [1]. The transition from carbon to tungsten was realized by coating fine grain graphite with tungsten of different thickness [2].

The next step in the divertor improvement is the installation of a solid tungsten divertor made from powder metallurgy (PM) tungsten: This expands the operational range of ASDEX Upgrade. (i) It overcomes the problem of delamination of thick tungsten coatings in discharges with a peaked target heat load profile. [2] (ii) It avoids a frequent exchange of target plates with thin coatings that might be required due to the strong erosion in the outer divertor. (iii) It allows physics investigation such as erosion and deuterium retention of solid tungsten. In addition, effects of castellation and target tilting can be investigated under reactor like magnetic configurations.

This paper presents the requirements and the principle design of the new divertor in section 2 . The concept validation as a combination of finite element (FE) analysis and high heat flux testing to ensure a reliable operation are presented in section 3 . The divertor modification is used to increase the pumping efficiency below the roof baffle and in the main chamber. The expected effects on the scrape-off layer (SOL) plasma parameters are modeled by SOLPS/Eirene and are presented in section 4. Finally, a summary is given.

\section{Status and design requirements}

ASDEX Upgrade is equipped with an adiabatically loaded divertor consisting of 16 sections with 8 target tiles each. The operational limits are set by the thermal properties of the material and the connection to the cooling structure as indicated in Fig. 1. For AUG with a low heat transfer coefficient into the cooling structure, the operational limits are the energy limit and the energy impact. The energy impact is set by the tolerable surface temperature during the discharge whereas the energy limit is given by the equilibrium temperature of the bulk that is usually reached a few tens of seconds after the end of the discharge.

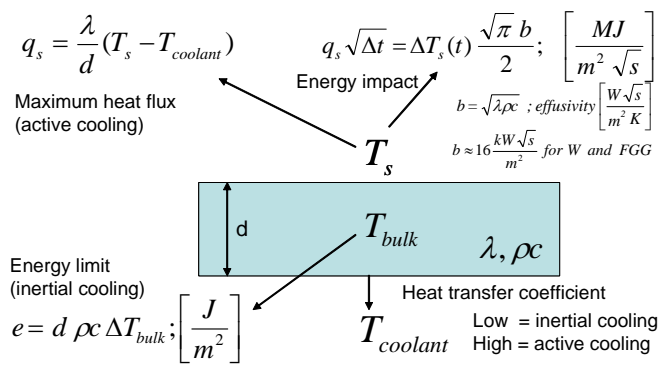

Fig. 1 Thermal simplified diagram of a divertor target with the energy impact, the maximum tolerated heat flux and the energy receiving capability as limiting figures.

In the case of $\mathrm{W}$ coatings, the maximum tolerable surface temperature depends on the mismatch of thermal properties of the coating and the bulk material. High surface temperatures and temperature gradients result in mechanical strain at the interface between the bulk material and the coating and have caused the delamination of the $200 \mu \mathrm{m}$ thick W-coating [2]. Uncoated graphite or solid tungsten allows a significantly higher surface temperature, i.e. energy impact. The energy receiving capability of coated and 
uncoated targets is comparable and depends on the heat capacity. It is about $50 \mathrm{MJ}$ for the outer divertor of AUG with $3 \mathrm{~cm}$ thick graphite tiles or about $150 \mathrm{MJ}$ plasma heating energy (details are discussed in [3]) .

These figures have to be compared to typical values for applied heating energy of about $50 \mathrm{MJ}$ in improved $\mathrm{H}$-mode discharges or about $60 \mathrm{MJ}$ in discharges with high $\mathrm{P} / \mathrm{R}$ values, i.e. discharges with maximum heating power. The applied heating energy during plasma discharges is usually below the design limit of the target mainly because of the fact that at high heating powers plasma instabilities are dominating the plasma performance and the stability limit is reached often already at 7-10 MW heating power.

From these thermal requirements follow that a new divertor should have a higher heat impact capability compared to the $\mathrm{W}$-coated divertor without the need to increase the energy receiving capability.

A design concept for a solid tungsten divertor was developed and tested in 2010. It consists of a sandwich structure that is compatible to the existing divertor support structure. The $30 \mathrm{~mm}$ thick sandwich is made from $15 \mathrm{~mm}$ thick tungsten in top of a $15 \mathrm{~mm}$ thick graphite adapter tile as presented in [3].

The concept to use flat solid tungsten plates with 15 $\mathrm{mm}$ thickness as target tiles was first tested in the high heat flux test facility GLADIS [4] and is now in operation in ASDEX Upgrade for 2 campaigns or about 1600 discharges [3].

Based on the successful test of the design concept the basic requirements for a new solid tungsten divertor Div-III were defined: (i) It should have the same energy and heat load receiving capability as a graphite divertor without tungsten coating. (ii) At present, the target plates are mounted to the support/cooling structure outside the vessel and the assembled divertor module is then installed into the torus. The use of solid tungsten tiles results in an increased weight of about 50 $\mathrm{kg}$ per divertor module. This requires tile assembly inside the vessel to keep the manual handling capability. (iii) The redesign of the divertor should be used to increase the pumping efficiency in the outer SOL by increasing the conductance between the divertor region and the cryo-pump, hopefully resulting in a decrease of the edge density. (iv) A reasonable price and the potential to be realized in the near future. The requirements (i)-(iii) are discussed more in detail in the following paragraphs.

(i) In between discharges, the divertor is cooled down to room temperature. During the discharge, the surface and the bulk temperature of the target tiles are permanently increasing, i.e. no equilibrium between heating and cooling is reached as typically for adiabatically loaded components. As a consequence, the surface temperature can increase up to the melting or sublimation temperature of the target material (Fig. 2). This large range of temperature evolution is not critical for graphite but means for tungsten that the temperature evolution starts with brittle material going through the temperature range where the material becomes ductile and a small fraction of the target thickness might reach temperatures above the limit for recrystallization of about $1500 \mathrm{~K}$. This is accepted for
ASDEX Upgrade because the total time with local target temperatures above $1300 \mathrm{~K}$ is about $50 \mathrm{~s} / \mathrm{a}$. The expected level of recrystallization is either negligible or limited to the first millimeters on top of the target. Even for the design case of $15 \mathrm{MW} / \mathrm{m}^{2}$ for $10 \mathrm{~s}$, the total time at temperatures above $1300 \mathrm{~K}$ is a few tens of seconds per year or about 10 shots. The maximum equilibrium temperature of the target that gives together with the heat capacity the energy receiving capability is specified with $1000^{\circ} \mathrm{C}$, i.e. it is well below the start temperature for recrystallization (see e.g. [5]).

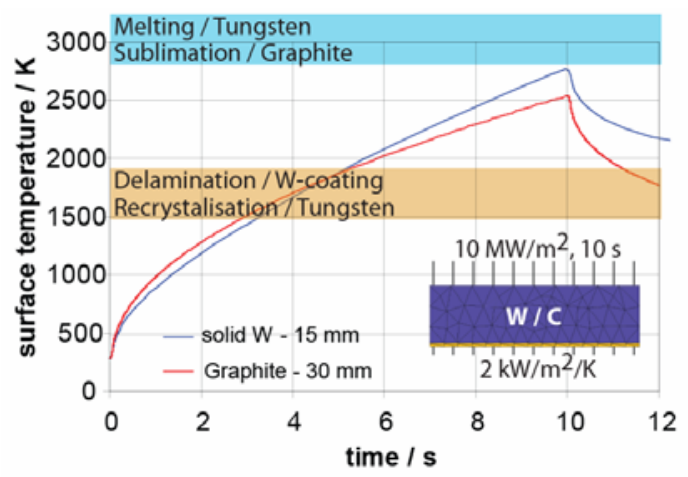

Fig. 2 Surface temperature evolution for a fine grain graphite and a solid tungsten target. The temperatures are calculated with a 2D-FEM code applying temperature dependent thermal parameters. Both targets are clamped with a graphite interlayer to the cooling structure realizing a weakly cooled target.

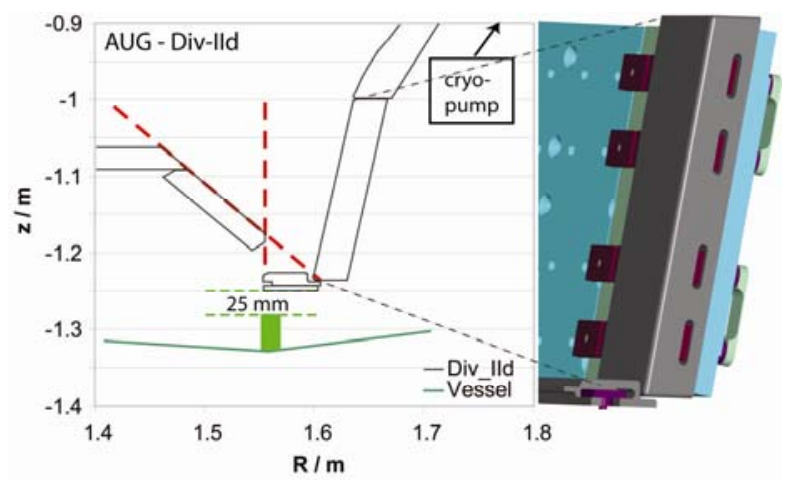

Fig. 3 Divertor geometry for the present Div-IIb divertor that has to be compared to the new Div-III geometry as shown in Fig. 4. Div-IId consists of $3 \mathrm{~cm}$ thick fine grain graphite plates clamped to a stainless steel cooling structure. The conductance below the outer divertor is low due to the small gab between divertor and vessel.

(ii) The present concept of target tile fixing to the cooling structure is based on sliding blocks inside the target clamped by spring dishes to the support structure. This concept requires a pre assembly outside the torus. In addition, it weakens the material thickness in the regions of the cuts for the gliding blocks (see Fig. 3). The new clamping concept as shown in Fig. 4, uses claws at the top and bottom side of the target. These claws are in regions with low heat load away from a possible separatrix position. The target tiles will be mounted inside the torus onto the pre-installed divertor structure by placing it onto the loose lower claw. Then the upper claw is mounted and both claws are strained resulting in a pre-load of $4 \times 6 \mathrm{kN}$ to each target tile. To realize a minimum pre-tension in the 
emergency case, the 4 screws for the pre-load are working against spring dishes with a force of $600 \mathrm{~N}$ each.

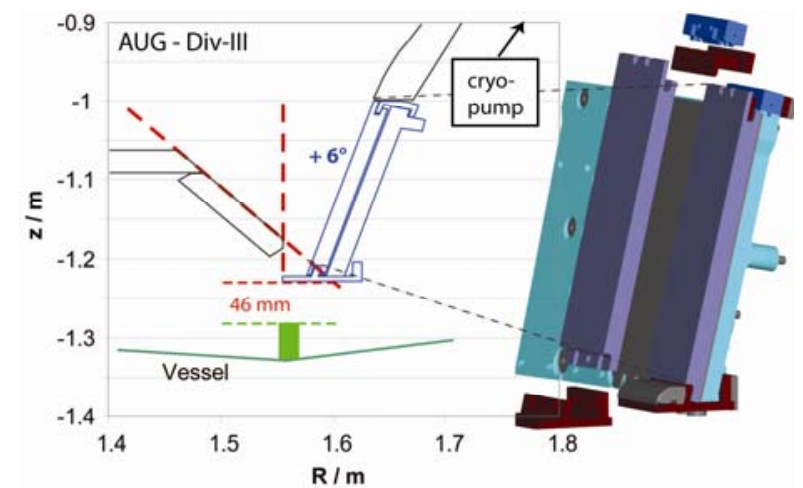

Fig. 4 Geometry of the new Div-III divertor. The target is shortened to increase the conductance by increasing the gap between divertor and vessel. This requires an inclination of the target by $6^{\circ}$ to allow the same magnetic configurations as in Div-IIb. Compare to Fig. 3 The exploded view of the target clamping shows the target, the upper and lower claw as well as the fixing of the upper claw.

(iii) The main vessel is pumped by a cryo-pump with about $150 \mathrm{~m}^{3} / \mathrm{s}$ pumping speed placed behind the outer divertor structure which reduces the effective pumping speed below the roof baffle and in the main chamber by about a factor of 5 . An increase of the effective pumping speed requires to increase the conductance below the outer divertor, i.e. to increase the gap between the lower part of the outer divertor and the vacuum vessel. Moving up the lower part of the outer divertor without a change of the target inclination would reduce the operational space for magnetic configurations.

The present divertor is magnetically tight for configurations with the outer separatrix located in the indicated range (Fig. 3). This range should be kept also for the new divertor because a significant fraction of magnetic configurations is using the lower third of the outer target. The resulting new target geometry is shown in Fig. 4. The gap between divertor and vessel is increased by $21 \mathrm{~mm}$ or about $80 \%$ by shortening the target and its support structure and increasing the target inclination from $9^{\circ}$ to $15^{\circ}$. The increase of the target heat load as a result of the increased target inclination is between 2 and $6 \%$ depending on the magnetic configuration. Consequences of the geometric modification onto the edge plasma are discussed in section 4.

\section{Concept validation by FE-analysis and high heat load tests.}

During the design phase for the Div-III target tiles details of the geometry were investigated with respect to their handling and assembling. In parallel the thermo-mechanical properties were investigated with 3D FE-Analysis (ANSYS). The final design of the target was optimized based on several FE-analysis and constructive adaptations to the existing divertor support and cooling structure. The FE-analysis reveals three main features: (i) Due to the fact, that the maximum heat load during discharges is deposited in the lower part of the target the lower clamping structure heats up to $80^{\circ} \mathrm{C}$. Because the target fixation is realized by a system of plates and pre-stressed screws, the temperature increase has to be taken into account by the design of the fastening system and the estimation of the force pre-stress at the screws. (ii) The maximum stress of about $680 \mathrm{MPa}$ in the area of the maximum heat load $\left(15 \mathrm{MW} / \mathrm{m}^{2}\right.$ for $\left.3 \mathrm{~s}\right)$ is above the Yield Strength of about $500 \mathrm{MPa}$ and reveals on a plastic deformation (see [6]) . The plastic deformation would be stretched in toroidal direction over about $10 \mathrm{~mm}$ and $6 \mathrm{~mm}$ depth. However, the high heat load tests in GLADIS have not shown any cracks due to plastic deformation. (iii) The mechanical stress induced in the clamping region is about $80 \mathrm{MPa}$ and well below any critical value Fig. 5.

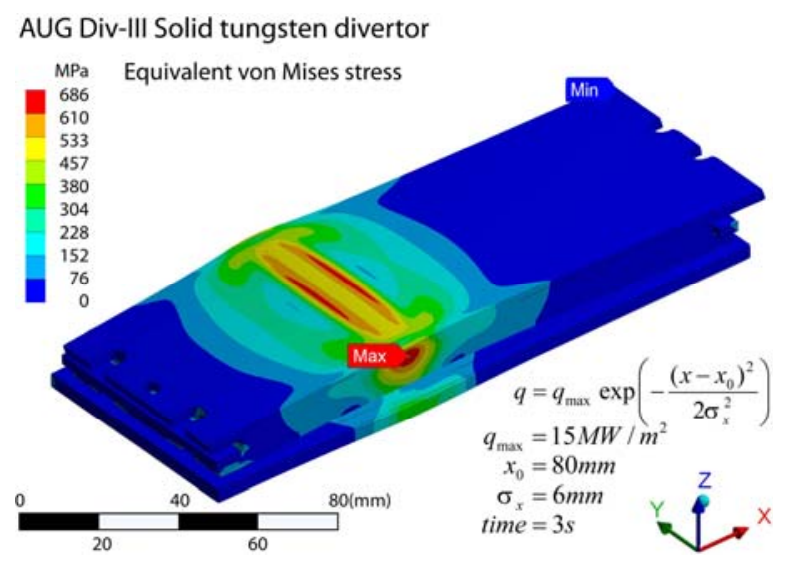

Fig. 5 Thermo-mechanical FE-analysis - von Mises stress distribution in the target.

The optimized design was then tested in GLADIS to validate the FE-analysis and to test out the load capacity and reliability of the target design. Four prototype tiles were ordered from two manufacturers and tested together with the clamping concept. One tile from each manufacturer was tested with the 'standard' qualification procedure. The other two tiles were tested with overload by increasing the heating power and energy to reach the surface temperature and to exceed the energy limit. Details on FE-analysis and GLADIS tests can be found in [6].

The standard test procedure for target qualification consists of 200 cycles with $10.5 \mathrm{MW} / \mathrm{m}^{2}$ for $3.5 \mathrm{~s}$. The corresponding integrated load to the target is $105 \mathrm{~kW}$ resulting in $367.5 \mathrm{~kJ}$. Extrapolated to the whole divertor, consisting of 128 tiles, the total divertor load is $13.5 \mathrm{MW}$ or $47 \mathrm{MJ}$, respectively. The standard qualification procedure represents discharges exploiting the energy receiving capability of $48 \mathrm{MJ}$ of the present divertor and the new Div-III and represents high power shots of more than 3 experimental campaigns of AUG. Both targets passed by the test without any sign of damage or surface modification.

In a next step, the heat load was increased to 23.5 MW/m $\mathrm{m}^{2}$ and $30 \mathrm{MW} / \mathrm{m}^{2}$ with a stepwise increase of the pulse duration up to $3 \mathrm{~s}$ and $2.8 \mathrm{~s}$, respectively. Consequently, the corresponding maximum energy input was $77 \mathrm{MJ}$ and $100 \mathrm{MJ}$, respectively, or about twice the design value for the energy receiving capability of the outer divertor. The corresponding 
surface temperatures values at the end of the heating pulse were $2800^{\circ} \mathrm{C}$ and $3300^{\circ} \mathrm{C}$. Finally, one tile was used for an overloading with 10 pulses $30 \mathrm{MW} / \mathrm{m}^{2}$ for $2.5 \mathrm{~s}$ followed by 30 pulses with 'standard' load of $10.5 \mathrm{MW} / \mathrm{m}^{2}$ for $3.5 \mathrm{~s}$.

All 4 tested tiles show no failure during or after the high heat load tests. No cracks due to plastic deformation were found at the surface. The overloaded tile shows a modification of the surface due to recrystallization and grain growth. In addition a small crack was found in the outermost edge of the fixing guide. The fact, that in contrast to the sandwich tiles no cracks were found at the surface of the tiles is attributed to the clamping with the claws on top and bottom in the less loaded regions. This clamping allows the target to deform during the heating phase and to relax afterwards. This was measured in detail by a high resolution CCD system and displacement sensors at the rear-side of the target. The deformation during the standard load case is $0.6+-0.1 \mathrm{~mm}$. This deformation is reversible, no plastic deformation $(<0.05 \mathrm{~mm})$ is found at the end of the qualification cycle. In contrast, the overloaded tiles show a stronger deformation during the heating pulse and a permanent deformation. It is about $1 \mathrm{~mm}$ for the overloaded tile.

The target deformation during the discharge is no real concern, because all tiles are deformed in the same direction and with the same amplitude as long as the divertor is symmetric. This is now realized with Div-III where all target tiles have the same tilting angle and shadowing depth in contrast to the present divertor that has twofold symmetry due to different toroidal gap widths. Such a difference in target tilting results in a periodic target load and adjacent tiles are receiving different loads [7]. Nevertheless, also differences in the target tilting can be tolerated by Div-III because the design shadowing depth is $0.8 \mathrm{~mm}$, i.e. larger than the maximum measured deformation for the design heat load.

The surface morphology of tiles exposed to GLADIS and AUG were investigated with metallography and SEM to quantify the recrystallization. Examples for metallographic micrographs are shown in Fig. 6. It is obvious that the grain size is increased for the overloaded tile that has experienced surface temperatures up to $3300^{\circ} \mathrm{C}$, whereas the AUG sample shows an increased grain size in the surface region only.

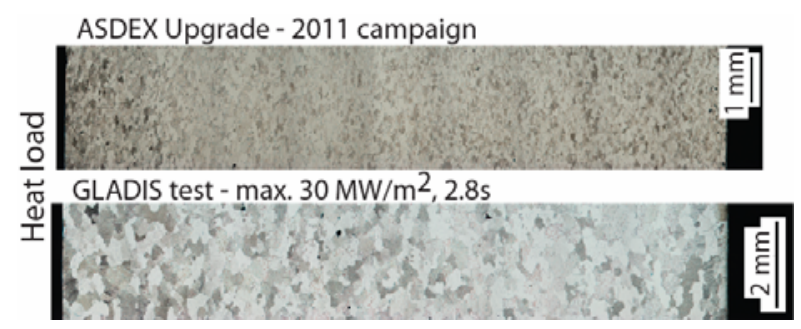

Fig. 6 Metallographic micrographs of targets with different heat load history. Top - target cross-section after one AUG experimental campaign with moderate grain growth near to the loaded surface; Bottom - cross-section of the overloaded target $\left(30 \mathrm{MW} / \mathrm{m}^{2}, 2.8 \mathrm{~s}\right)$ showing an increased grain size over nearly half of the thickness due to recrystallization.

\section{Pumping and SOL}

SOLPS5.0 and EIRENE (version 2001) modeling was used to assess the impact of the divertor geometry change on pumping efficiency, plasma performance and operational space. A well characterized discharge from carbon migration experiments was used to compare the two divertor geometries [8].

In the modeling the separatrix density and the power into the SOL were kept constant $\left(\mathrm{n}_{\mathrm{e}, \mathrm{sep}}=\right.$ $1.1 \times 10^{19} \mathrm{~m}^{-3}$ and $\left.\mathrm{P}_{\mathrm{SOL}}=2 \times 0.35 \mathrm{MW}\right)$. The modeled mid-plane profiles of density and temperature were virtually the same, whereas the target density was decreasing and consequently the target electron temperature was increasing by about 5-10\%. Due to the enhanced pumping efficiency of the Div-III geometry the required mid-plane puff rate had to be increased by about $20 \%$. Here, the pumping efficiency was defined as the ratio of neutral fluxes in the pumping chamber and below the roof baffle. It is increased by about $30 \%$ with the new geometry. These findings are an indication for an easier access to lower SOL densities as expected for a higher pumping efficiency. The option to operate at high edge densities is regained by a modification of the cryo pump so that it can be used fractional.

\section{Summary and Conclusions}

Div-III, a divertor with solid tungsten target tiles at the outer strike line is designed and tested and will be installed in 2013. It is a further step in exploring tungsten as material for plasma facing components. It avoids the restrictions of tungsten coatings on graphite and realizes an operation range up to $50 \mathrm{MJ}$ energy removing capability in the outer divertor. In addition, it allows physics investigation such as erosion and deuterium retention as well as effects of castellation and target tilting.

The design of the target itself and the clamping was optimized with FE-analysis and was intensively tested with 4 prototype tiles from 2 manufacturers up to a double overload in GLADIS. Cyclic tests reveal that the target and the clamping structure can be operated with the design load of $50 \mathrm{MJ}$ without any damage. A double overload of the target results in local recrystallization and minor cracks but also these damaged targets did not fail during operation. This is supported by sandwich test tiles that were operated in AUG for 2 campaigns. These tiles show no failure despite two small cracks in front of the mounting wholes that are eliminated by the new design.

The necessary adaptation of the divertor structure to the modified clamping concept was used simultaneously to increase the conductance between the cryo-pump and the divertor region. The impact of the changed pumping efficiency was investigated with SOLPS/Eirene modeling. The modelling results are an indication for an easier access to lower SOL densities as expected for a higher pumping efficiency in the main chamber.

\section{References}

[1] R. Neu, et al., Phys. Scrip., T138 (2009) 014038. 
[2] A. Herrmann, et al., Phys. Scrip., T138 (2009) 014059.

[3] A. Herrmann, et al., Phys. Scrip., T145 (2011) 014068.

[4] H. Greuner, et al., J. Nucl. Mater., 367-370 (2007) 14441448.

[5] P. Mertens, et al., Phys. Scrip., T138 (2009) 014032.

[6] N. Jaksic, et al., This conference (SOFT), (2012).

[7] A. Herrmann, et al., Fusion Eng. Des., 86 (2011) 530-

534.

[8] L. Aho-Mantila, et al., J. Nucl. Mater., 415 S231-S234. 